\title{
Anti-icing Properties on Surfaces through a Functional Composite: Effect of Ionic Salts
}

\author{
Derya Aydin, ${ }^{\dagger}$ M. Birgul Akolpoglu, ${ }^{\dagger}$ Riza Kizilel, ${ }^{\dagger}$ and Seda Kizilel ${ }^{* \dagger, \ddagger \odot}$ \\ ${ }^{\dagger}$ Department of Chemical and Biological Engineering and ${ }^{\ddagger}$ Koç University-TÜPRAŞ Energy Center (KUTEM), Koc University, \\ Sariyer, Istanbul 34450, Turkey
}

\section{Supporting Information}

\begin{abstract}
This study reports the potential of a unique functional composite for anti-icing applications. To date, various ionic salt formulations have been applied to prevent ice accumulation on surfaces. However, salt can be removed by external factors and large amounts must be used to attain anti-icing properties. Incorporating hydrophilic salts into hydrophobic mediums and controlled release of specific agents can provide effective solution to reduce ice
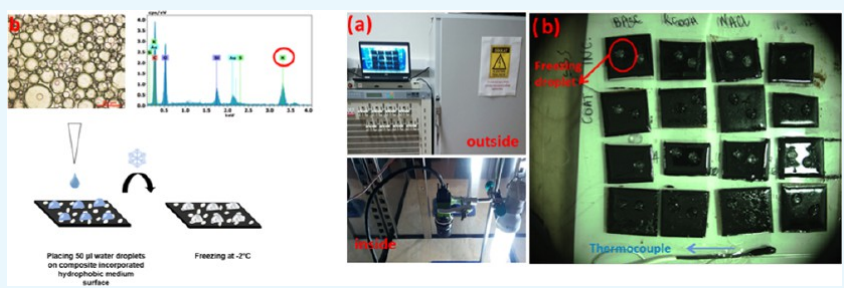
accumulation on surfaces. Here, we developed functional polymer composites with salt pockets of altered ionic salts consisting of potassium formate $(\mathrm{KCOOH})$, sodium chloride $(\mathrm{NaCl})$, or magnesium chloride $\left(\mathrm{MgCl}_{2}\right)$. We dissolved ionic salts in hydrophilic gel domains and dispersed in a hydrophobic styrene-butadiene-styrene polymer matrix. $\mathrm{Na}^{+}$and $\mathrm{Cl}^{-}$ions delayed ice formation by $42.6 \mathrm{~min}$ at $-2{ }^{\circ} \mathrm{C}$ compared to that for unmodified surfaces. Functional composites prepared with the $\mathrm{NaCl}$ ionic salt exhibited better anti-icing behavior at $-2{ }^{\circ} \mathrm{C}$ because of their high concentration compared to that of the composites prepared with $\mathrm{KCOOH}$ and $\mathrm{MgCl}_{2}$ ionic salts. We also characterized the release of ionic salts from composite-modified hydrophobic medium separately up to 118 days. Furthermore, we monitored freezing of water on composite-incorporated or composite-coated hydrophobic surfaces in a camera-integrated cold chamber with a uniform temperature $\left(-2{ }^{\circ} \mathrm{C}\right)$. The results demonstrated significant increases in the delay of freezing on composite-incorporated or composite-coated surfaces compared to that on controls. We observed altered effects of each ionic salt on the mechanical, morphological, and functional properties of the composite-incorporated or composite-coated hydrophobic surfaces. Our results suggested that the efficiency of a polymer composite to promote anti-icing behavior on a surface is directly related to the type and concentration of the particular ionic salt incorporation into the composite. This approach is promising and demonstrates significant potential of the ionic salt embedded within polymer composite-modified hydrophobic surfaces to attain delayed icing function.
\end{abstract}

\section{INTRODUCTION}

Anti-icing strategies are based on disrupting ice crystallization before it builds up on a surface. Ionic salts can depress the freezing point of water and therefore they have been commonly used to eliminate accumulation of ice on surfaces. Yet, salt can easily be removed by external factors and requires frequent application. Besides their economical consideration, using large amounts of anti-icing agents weakens mechanical properties of surfaces and has negative impacts on living organisms and the environment. ${ }^{1-3}$

Sodium chloride $(\mathrm{NaCl})$, calcium chloride $\left(\mathrm{CaCl}_{2}\right)$, potassium formate $(\mathrm{KCOOH})$, and magnesium chloride $\left(\mathrm{MgCl}_{2}\right)$ have been frequently used for this purpose. ${ }^{1}$ However, bulk application of salts on surfaces has negative impact on the mechanical strength of the underlying surface and damages vehicles, plants, and many living organisms. To address this limitation, several other approaches were used to eliminate ice upon its formation on a surface. Electrical systems have been considered through the use of wiring underneath surfaces and activation of heating when needed to reduce ice deposition. However, this application has high energy requirements and demands high costs and hence considered as not feasible. Because of these limitations associated with deicing, many studies focused on the development of an antiicing approach through delaying of ice formation on surfaces. $^{2-4}$

Altering the topology is a major strategy to achieve an icerepellent surface. Self-assembled monolayers, bulk coatings, micro- and nanostructures, and infused polymers can be employed on surfaces for the development of anti-icing property, and a common method to reduce ice adhesion and deposition involves super-hydrophobic surfaces. ${ }^{2,4-18}$ This approach has shown several promising results and is based on the rough surface structure to inhibit nucleation of ice on a variety of surfaces. However, the super-hydrophobic surface approach is not always applicable for all surfaces as those surfaces promote slip with exposure to water. ${ }^{10,19}$ These surfaces also result in a high ice adhesion strength on rough surfaces because of the large contact area between the solid and the ice under high humidity and water condensation

Received: April 25, 2018

Accepted: July 2, 2018

Published: July 17, 2018 


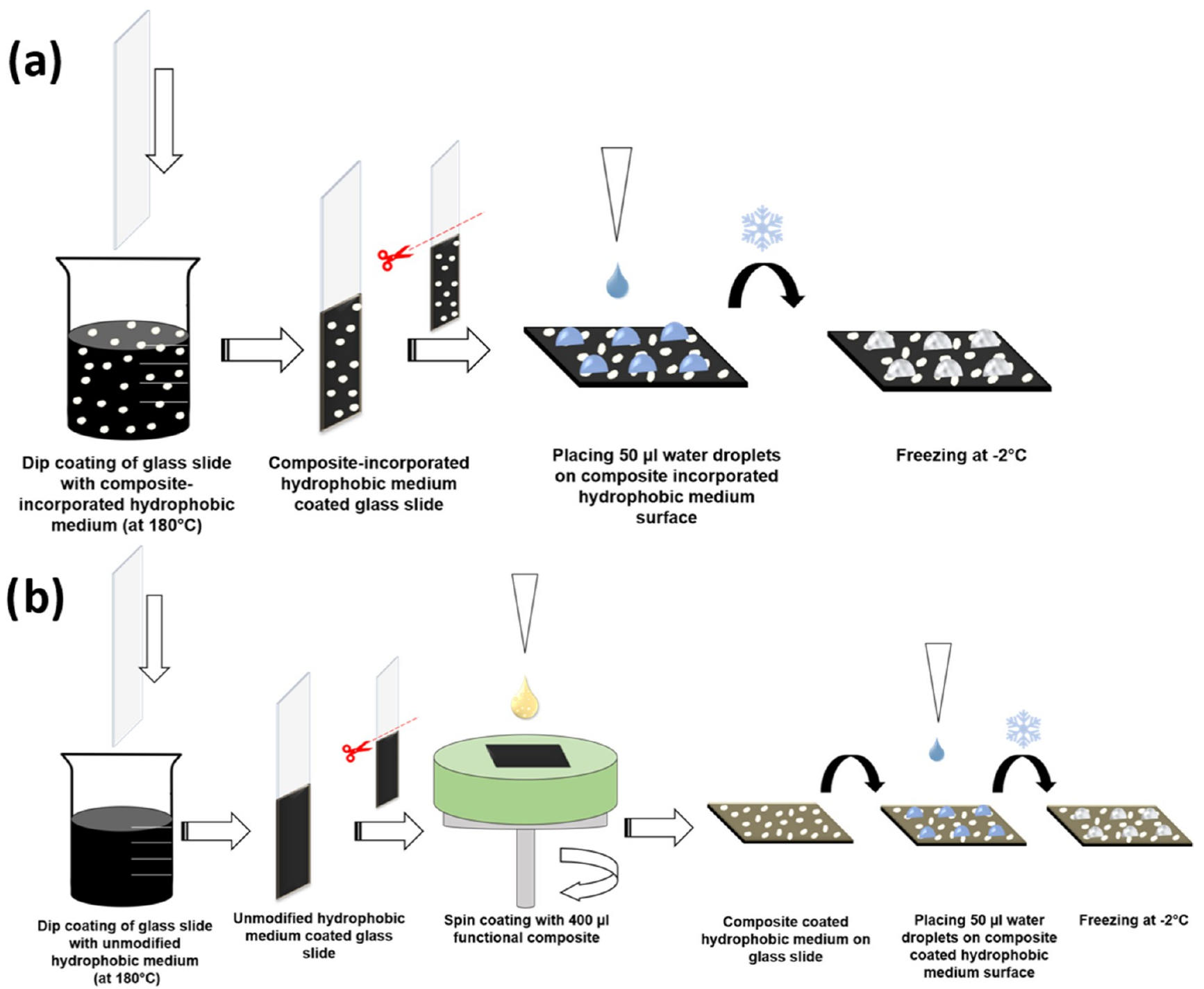

Figure 1. Schematics for the preparation of (a) composite-incorporating and (b) composite-coated samples.

conditions. ${ }^{20}$ In addition, repeated icing/deicing cycles on super-hydrophobic surfaces damage the micro/nanostructure, which further increases ice adhesion strength with time. ${ }^{21}$

Other approaches to promote anti-icing on surfaces included graphite and carbon fiber-reinforced conductive structure ${ }^{5}$ and rubber nanoparticles. ${ }^{6}$ Geothermal energy was also used to delay icing on tunnels and bridges. ${ }^{7,22}$ Feasibility of superhydrophilic polymer brush coatings, ${ }^{9}$ super-hydrophobic coatings on composite silicone rubber, ${ }^{8}$ nanoparticle-polymer composites, ${ }^{23}$ and anti-icing fillers were investigated as alternative methods for anti-icing applications. ${ }^{3,24,25}$ In another study, researchers tested the potential of fillers such as $\mathrm{NaCl}$, calcium carbonate $\left(\mathrm{CaCO}_{3}\right)$, and magnesium carbonate $\left(\mathrm{MgCO}_{3}\right)$ into complex hydrophobic fluids to gain anti-icing property. Delays in frost formation and decreases in adhesion between the ice and surfaces were reported. However, these fillers included high concentrations of salt $(70-75 \%(w / w))$, which had negative impacts on the mechanical and morphological properties of the underlying surface.

To date, hydrophilic ionic salts could not be effectively incorporated into hydrophobic surfaces and controlled release of these molecules from surfaces to obtain anti-icing property has not been achieved. To address these challenges, we modified a hydrophobic medium with a polymer composite, which was prepared using the nanoparticle-stabilized Pickering emulsion approach. The polymer composite has a hybrid nature and contains anti-icing agents within the hydrophilic domains that are embedded in a continuous hydrophobic styrene-butadiene-styrene (SBS) polymer. We investigated the mechanical, morphological, and functional properties of modified hydrophobic platforms in our previous studies where we used potassium formate ionic salt as an anti-icing agent. ${ }^{26,27}$ Potassium formate has many advantages compared with other salts such as its high solubility and environmentally friendly properties. However, it is costly compared with the other commonly used $\mathrm{NaCl}, \mathrm{MgCl}_{2}$, or $\mathrm{CaCl}_{2}$.

In our current study, to further explore the influence of different hydrophilic ionic salts on the mechanical, morphological, and functional properties, we prepared hydrophobic surfaces that were either modified or coated with our composites. We investigated the potential of $\mathrm{NaCl}, \mathrm{MgCl}_{2}$, and $\mathrm{KCOOH}$ in our functional polymer composite, where we monitored freezing of water in realistic conditions. The results suggested that freezing delays could be tuned with the type and amount of hydrophilic ionic salt incorporation into the composite as well as through the surface coating strategy. 
According to our knowledge, it is the first time that such welldefined surface characterizations have been systematically investigated through functional hybrid composites to promote the anti-icing property. This approach is promising for the development of complex hydrophobic fluids and hence surfaces are prepared with these fluids, with the anti-icing function and for industrially relevant applications.

\section{MATERIALS AND METHODS}

2.1. Materials. Styrene-butadiene-styrene (SBS, S/B weight fraction: 70:30) block copolymer (Kraton D1101), potassium formate $(\mathrm{KCOOH})(99 \%)$, magnesium chloride $\left(\mathrm{MgCl}_{2}\right)$ (99\%) and $\mathrm{NaCl}$ (99\%) (Sigma-Aldrich), silica nanoparticles (AEROSIL R 816), agar powder (SigmaAldrich), and cyclohexanone (99.9\%) (Merck) were used for the synthesis of particle-stabilized emulsions. The hydrophobic fluid (50/70 penetration grade bitumen) was kindly provided by TÜPRAŞ. Deionized water was used in all experiments (PURELAB Option, ELGA).

2.2. Preparation of Composite-Modified Hydrophobic Surfaces. 2.2.1. Preparation of Pickering Emulsions. Emulsions were prepared as described in our previous study. ${ }^{26}$ Briefly, emulsions with $0.25(\mathrm{v} / \mathrm{v})$ internal volume fraction $(\Phi$ $=0.25)$ and $1.5 \%(\mathrm{w} / \mathrm{v})$ nanoparticle concentration in the internal phase were prepared. The concentration of salt used in emulsions was determined on the basis of the solubility of $\mathrm{NaCl}$ in water because $\mathrm{NaCl}$ has the lowest solubility in water among the salts used in this work. Thus, $0.5 \mathrm{~mL}$ of ionic salt $(0.3 \mathrm{~g} / \mathrm{mL})$ salt solution-dissolved agar was added to $0.5 \mathrm{~mL}$ of $1.0 \%(\mathrm{w} / \mathrm{v})$ nanoparticle-cyclohexanone stock solution under mixing by vortex for $10-15 \mathrm{~s}$. After the formation of Pickering emulsion, $1 \mathrm{~mL}$ of the SBS polymer in cyclohexanone (110 $\mathrm{mg} / \mathrm{mL}$ ) was added drop-wise to $1 \mathrm{~mL}$ of the ionic salt, agar, and nanoparticle mixture by mixing gently.

2.2.2. Preparation of Functional Composites and Incorporation into the Hydrophobic Fluid. Prepared emulsions were cast in Petri dishes to obtain functional composite membranes. Then, dry membranes were incorporated into the hydrophobic fluid $(5 \%(\mathrm{w} / \mathrm{v}))$, as described in our previous study. ${ }^{26,27}$ After processing, the samples were kept at room temperature. These samples are referred to as composite-incorporated surfaces throughout the text.

2.2.3. Preparation of Composite-Coated Hydrophobic Surfaces. Emulsions were coated on hydrophobic surfaces via spin-coating (Figure 1). Five hundred microliter of the emulsion was dropped on $4 \mathrm{~cm}^{2}$ of the hydrophobic fluidcovered surface and coated with a spin-coater (SCS-Special Spin-Coating Systems) at $1000 \mathrm{rpm}$ for $60 \mathrm{~s}$. Next, emulsioncoated slides were dried overnight to obtain composite coatings on hydrophobic surfaces. These samples are referred to as composite-coated surfaces throughout the text.

2.3. Surface Characterization of Composite-Modified Surfaces. 2.3.1. Fluorescence Microscopy. Fluorescence microscopy was used to infer SBS distribution in polymermodified hydrophobic surfaces. The morphologies of composite-incorporated hydrophobic fluids were analyzed under fluorescence microscopy with an emission wavelength of 520 $\mathrm{nm}$ and excitation wavelength of 450-490 nm.

2.3.2. Atomic Force Microscopy (AFM). Atomic force microscopy (AFM) experiments were conducted in a Bruker Dimension ICON AFM with MPP21100-10 REFSP tips. $20 \times$ $20 \mu \mathrm{m}^{2}$ of sample surfaces were scanned. Imaging was performed in soft tapping mode at room temperature to acquire phase difference microscopy images.

2.4. Dynamic Mechanical Analysis. The rheology experiments were performed using a Discovery Hybrid Series-2 Rheometer (TA). Dynamic mechanical analysis was conducted using oscillation tests between parallel plates within the region of the linear viscoelastic response. Viscoelastic parameters were measured at $0.02 \mathrm{~Hz}$ constant frequency and temperature sweeps between 20 and $120{ }^{\circ} \mathrm{C}$. The tests were undertaken with $20 \mathrm{~mm}$ diameter parallel plate geometry with a $1 \mathrm{~mm}$ gap. $^{26}$

2.5. Characterization of Functional Properties. 2.5.1. Salt Release Characterization. The release of $\mathrm{KCOOH}, \mathrm{NaCl}$, and $\mathrm{MgCl}_{2}$ from composite-modified surfaces was monitored by immersing the samples in water and measuring the $\mathrm{Cl}^{-}$and $\mathrm{K}^{+}$ions using ion-selective electrodes (ISE K 800 and $\mathrm{Cl} \mathrm{DIN).} \mathrm{Composite-incorporated} \mathrm{surfaces}$ were incubated in $12 \mathrm{~mL}$ of deionized water, and cumulative release of the $\mathrm{K}^{+}$and $\mathrm{Cl}^{-}$ions was measured for 118 days using ion-selective electrodes (WTW potassium combination electrode $\mathrm{K} 800$ and $\mathrm{Cl}$ DIN, respectively) at specific time points. The total amount of salt released was calculated from measured $\mathrm{Cl}^{-}$and $\mathrm{K}^{+}$concentrations. ${ }^{26}$

2.5.2. Characterization of Anti-icing Properties of Surfaces. Delays in the icing properties of samples were investigated through monitoring the duration of freezing of water on functional composite-incorporated and compositecoated surfaces separately. For the case of compositeincorporated samples, glass slides were dip-coated with the functional hydrophobic fluid, and for the coated samples, glass slides were dip-coated with the unmodified hydrophobic fluid, followed by spin-coating (SCS-Special Coating Systems) of $500 \mu \mathrm{L}$ of functional composite solution (prepared with $\mathrm{NaCl}$, $\mathrm{KCOOH}$, or $\mathrm{MgCl}_{2}$ ) at $1000 \mathrm{rpm}$ for $60 \mathrm{~s}$ (Figure 1). Water droplets $(50 \mu \mathrm{L})$ were placed onto the surface of all samples. Freezing of water droplets was induced in a temperaturecontrolled chamber (Pol-Eko-Apartura) at $-2{ }^{\circ} \mathrm{C}$. The freezing process was monitored by a color area scan camera (JAI) with high-resolution lens (KOWA). Disappearance of transparency in water droplets was considered as the time of freezing. Freezing tests on surfaces were performed twice with the same samples consecutively to elucidate the effects of the repeated freezing-thawing process on the icing property of surfaces. After the first freezing test, water droplets were kept on the surface and evaporated. After the surfaces were fully dry, new water drops were placed on surfaces for the second freezing test. Schematics for the preparation steps of compositeincorporated and composite-coated surfaces are illustrated in Figure 1.

2.5.3. Ice Adhesion Test. An ice adhesion test was performed to determine the effects of different salts on the adhesion strength. Water droplets $(50 \mu \mathrm{L})$ were placed on the surface of unmodified, coated, and polymer compositeincorporated samples and frozen. Next, samples were placed in a spin-coater (SCS-Special Spin-Coating Systems) and spun at $1000 \mathrm{rpm}$. Samples were examined to determine the time for the removal of ice from the surface with constant centrifugal force. Experiments were repeated at least four times, and the average duration of adhesion for each sample was recorded. 

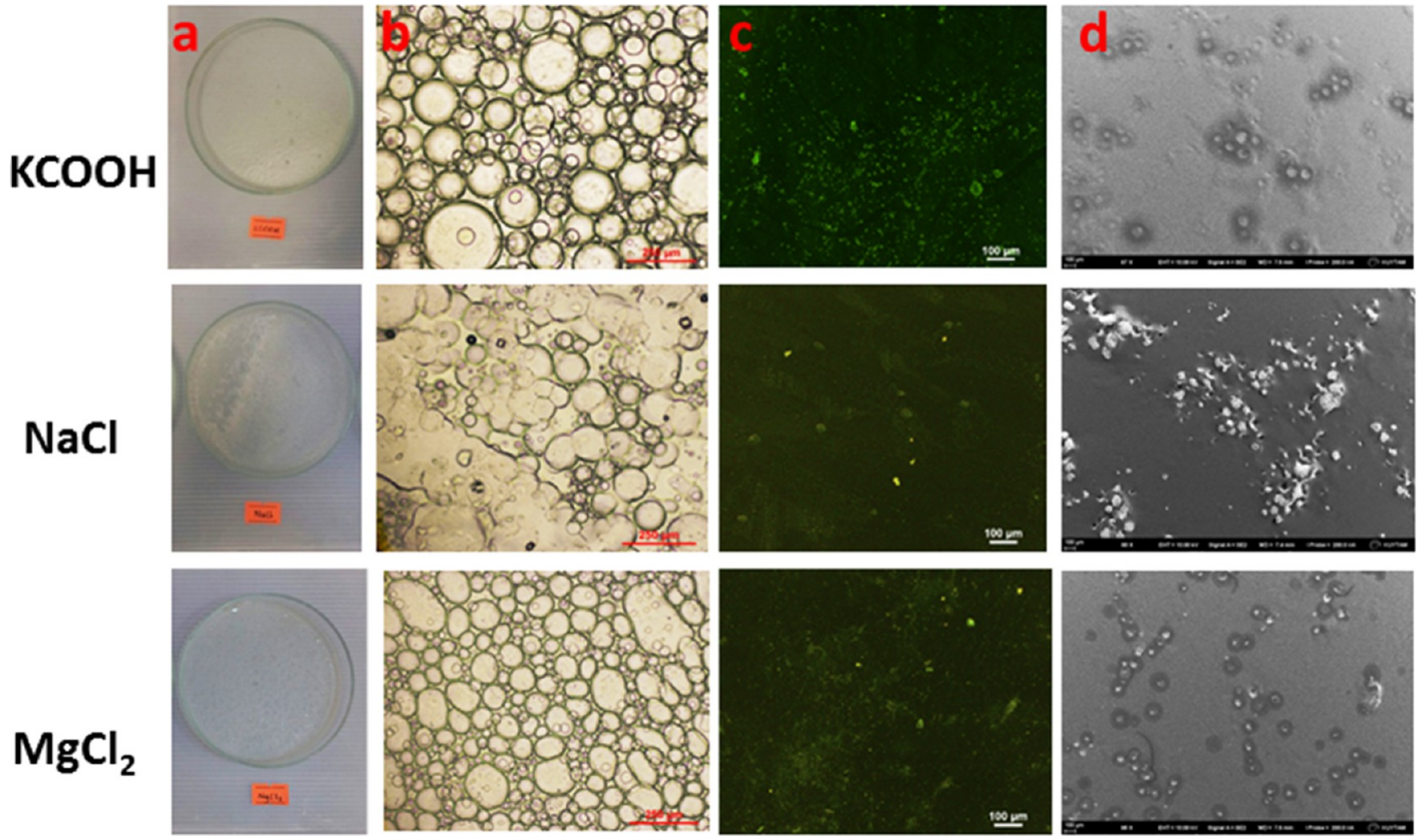

Figure 2. Images of composite membranes, emulsions, and composite-incorporated and composite-coated hydrophobic surfaces prepared using different salts. (a) Macroscopic appearance of composite membranes after emulsions are templated and dried on a surface, (b) microscopic image of emulsions in the wet state, (c) fluorescent microscope images of the composite-incorporated hydrophobic fluid, and (d) SEM images of composite-coated hydrophobic surfaces prepared with different ionic salts, scale bar $=100 \mu \mathrm{m}$.

\section{RESULTS AND DISCUSSION}

\subsection{Fluorescence, Light, and Scanning Electron} Microscopy (SEM) Characterization. Emulsions were prepared by dispersing the aqueous phase of $\mathrm{NaCl}, \mathrm{MgCl}_{2}$, and $\mathrm{KCOOH}$ in the continuous phase. Next, emulsions were casted on Petri dishes to obtain dry composite membranes. Composite-incorporated hydrophobic fluids were prepared with either incorporation of the composite within the hydrophobic fluid or coating of the hydrophobic surfaces with the composite. Fluorescence microscopy was used to infer the SBS morphology in composite-incorporated surfaces, whereas the surface morphology of composite-coated surfaces was investigated with SEM. The morphologies of SBS in composite-incorporated surfaces were investigated under fluorescence microscopy. The macroscopic appearance of composite membranes is demonstrated with the corresponding microscopic image of emulsions, fluorescence microscopy images of surfaces prepared with incorporation of the composite into the hydrophobic fluid, and SEM images of surfaces prepared with composite coating of hydrophobic surfaces in Figure 2. SEM images of the unmodified hydrophobic surface are demonstrated in Figure S1. We observed that emulsions prepared with $\mathrm{KCOOH}$ or $\mathrm{MgCl}_{2}$ are more stable and have more uniform morphology compared to those of the ones prepared with $\mathrm{NaCl}$. This difference resulted in the formation of uniform structures in composite membranes prepared with $\mathrm{KCOOH}$ or $\mathrm{MgCl}_{2}$. The difference could be attributed to the effects of high concentration of salt on emulsion stability, which was also reported in previous studies. ${ }^{28-30}$ For example, the effect of various ionic salts including $\mathrm{NaCl}, \mathrm{CaCl}_{2}, \mathrm{KCl}, \mathrm{NaCH}_{3} \mathrm{CO}_{2}$, and $\mathrm{NaNO}_{3}$ on water-seed oil emulsion stability were investigated, and decreases in the stability of emulsions as well as separation of water from oil were observed. ${ }^{28}$ In our study, the amount of mass used for each salt was kept constant and $\mathrm{NaCl}$ has the highest concentration among other salts because of its low molecular weight. Thus, the higher concentration of $\mathrm{NaCl}$ might have compromised the stability of emulsions. Another possible reason might be the negative influence of $\mathrm{Na}^{+}$ions on the agar gel network. In a previous study, sodium sulfate was shown to damage the integrity of agarose gel. ${ }^{29}$ In our study, high concentrations of $\mathrm{Na}^{+}$affected agar gel negatively and resulted in the formation of nonuniform structures in composite membranes. This result is also consistent with our SEM observations, where we observed spherical, gel-like, and embedded agar domains when surfaces were coated with $\mathrm{KCOOH}$ - or $\mathrm{MgCl}_{2}$-containing composites. These domains appeared as rigid in the $\mathrm{NaCl}$-containing composite, which could be due to insufficient gelation of agar at high concentration of sodium ions.

3.2. Energy-Dispersive X-ray (EDX) Characterization. Composite-coated hydrophobic surfaces were examined with EDX for elemental analysis. Spherelike domains observed on composite-coated hydrophobic surfaces were analyzed for the presence of salt. Both inside and outside of these domains were tracked for elemental analysis of the particular salt to confirm proper encapsulation of ionic salt within each domain. Figure 3 demonstrates EDX spectra of the inner and outer regions of the spherelike domains on hydrophobic surfaces. Those surfaces coated with emulsions of altered ionic salts were presented along with their corresponding SEM images. Figure 

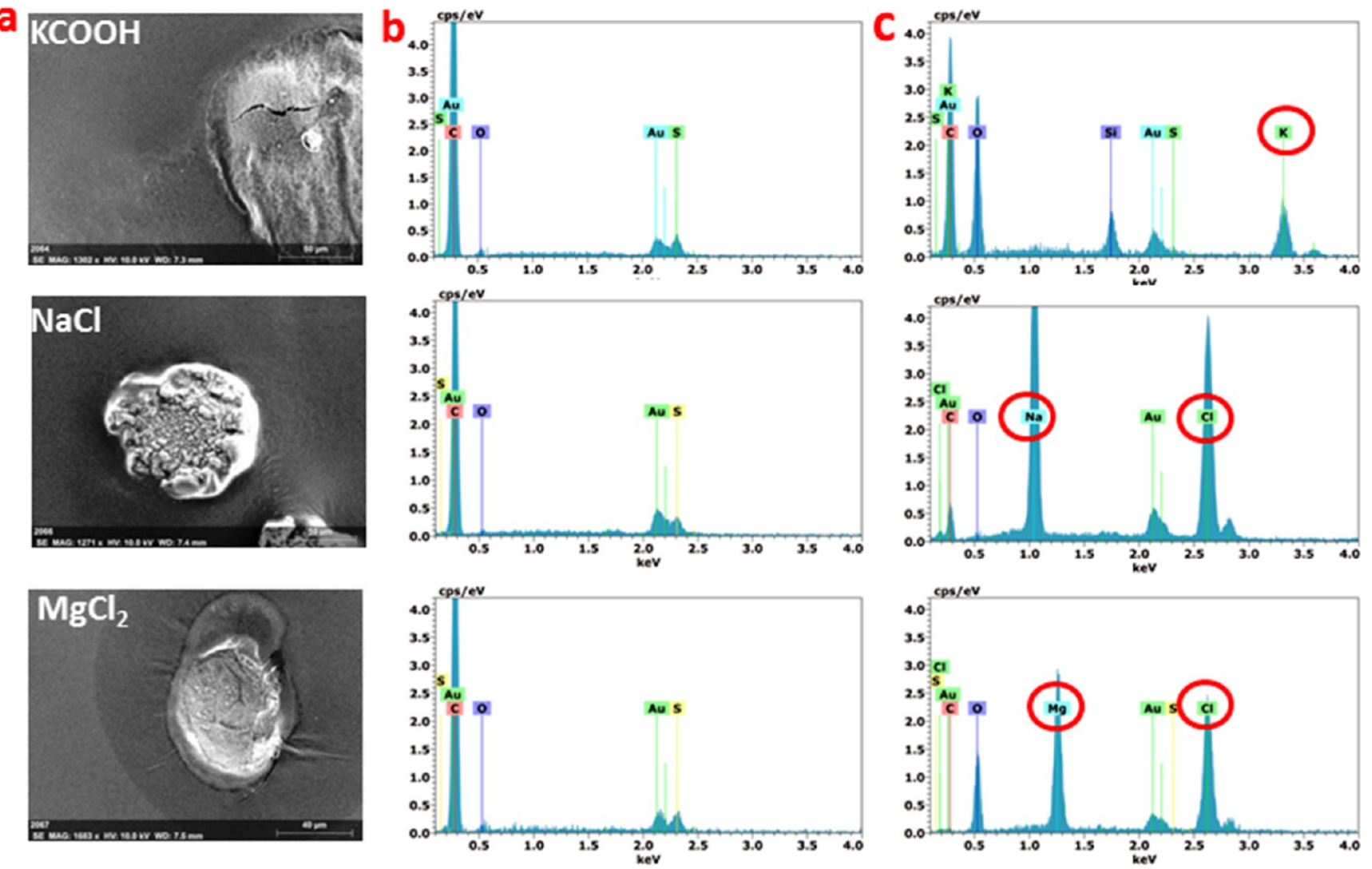

Figure 3. EDX analysis of hydrophobic surfaces coated with composites of different salts. (a) SEM images, (b) EDX spectra of the hydrophobic surface outside salt domains, and (c) EDX spectra of the hydrophobic surface inside salt domains. Red circles indicate the presence of particular elements.

3B,C includes EDX characterization from outside and inside ionic salt domains on hydrophobic surfaces, respectively. The peak found for the $\mathrm{K}^{+}$element on the $\mathrm{KCOOH}$ salt-containing composite-coated surfaces is an indication of the presence of $\mathrm{KCOOH}$ inside the spherelike domain. The fact that the $\mathrm{K}$ element was not detected outside the spherelike domains proved once more that ionic salt was only dissolved in the hydrophilic agar gel phase during the preparation of the emulsion and that spreading of salt toward uncoated regions of the surface did not occur. Similarly, $\mathrm{Na}^{+}$and $\mathrm{Cl}^{-}$were observed inside the spherelike domains for the $\mathrm{NaCl}$ containing composite-coated hydrophobic surface and $\mathrm{Mg}^{2+}$ and $\mathrm{Cl}^{-}$were detected for the $\mathrm{MgCl}_{2}$-containing compositecoated hydrophobic surface. These elements were not present outside the spherelike gel domains.

3.3. Atomic Force Microscopy Characterization of Surfaces. Atomic force microscopy (AFM) scans covered areas of $20 \times 20 \mu \mathrm{m}^{2}$ in hydrophobic surfaces that included composite incorporation or coating. Figure $4 \mathrm{~A}$ demonstrates phase images of surfaces prepared with the hydrophobic fluid, where two phases, continuous and dispersed phases, were observed. The other important feature of these hydrophobic surfaces is the appearance of "bee-shaped structures", which can be characterized with AFM microscopy. This bee-shaped feature was retained with the incorporation of altered ionic salts. These structures look like strings, which are immersed and emerged in the surface. The uniform distribution of these features confirmed that composite-modified hydrophobic surfaces had uniform structures. These structures also correspond to stiffer parts in the fluid and provide information about the mechanical properties. ${ }^{31,32}$ Bee-shaped structures were not observed when hydrophobic surfaces were coated with composites of altered ionic salts. This was expected, as bee-shaped features are attributed to the characteristic of hydrophobic fluid, and by coating, this feature becomes hidden underneath the coating (Figure 4B). Nonuniform structures were also observed on coated surfaces because of the differences in hydrophobicities of the surface and ionic salts.

3.4. Rheological Characterization of the CompositeModified Fluid. Dynamic mechanical tests were conducted to measure the viscoelastic properties of the unmodified and modified hydrophobic fluids. Oscillatory tests were used to measure the viscoelastic parameters, complex modulus $\left(G^{*}\right)$, and the phase angle $(\delta)$. $G^{*}$ provides information about the total resistance of a material to deformation under shear loading, whereas $\delta$ is a measure of viscoelastic balance of the material behavior. $\delta$ is the phase difference between the shear stress and the shear strain in an oscillatory test and gives information about the ratio of loss modulus $\left(G^{\prime \prime}\right)$ to storage modulus $\left(G^{\prime}\right) .^{33}$

Isochronal plots of the complex modulus, $G^{*}$, versus temperature at $0.02 \mathrm{~Hz}$ for unmodified and compositeincorporated hydrophobic fluids are presented in Figure 5A. According to Figure 5A, there is no significant difference between $G^{*}$ values of unmodified and composite-incorporated fluids at high temperatures. However, 1 order of magnitude difference was observed between the hydrophobic fluid modified with the $\mathrm{NaCl}$-added composite and the other groups at low temperatures. This difference is an evidence for a minor decrease in the resistance of the fluid at low 

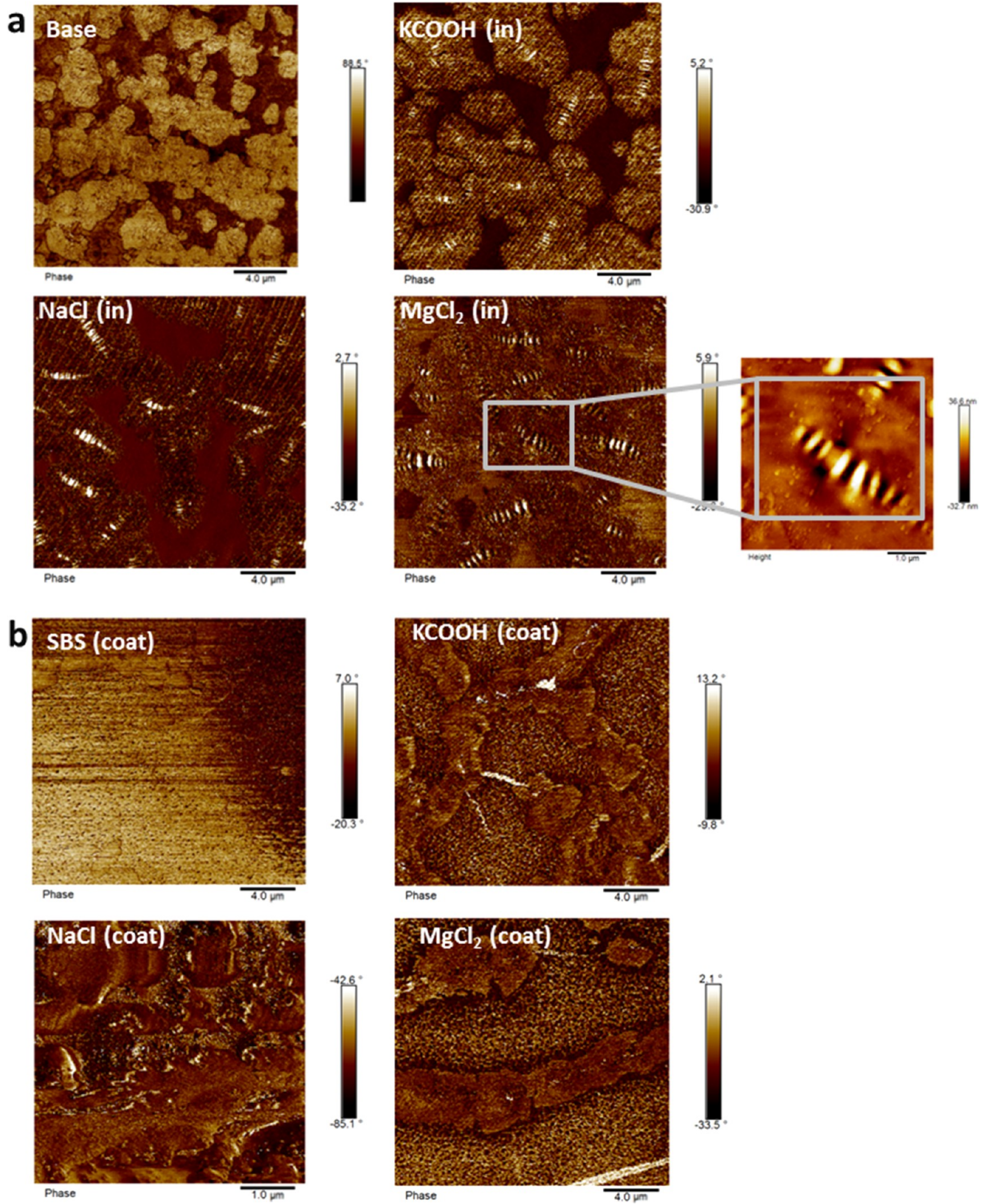

Figure 4. AFM phase difference images of (a) unmodified hydrophobic surface (base) and surfaces prepared with different salt compositeincorporating hydrophobic fluids, the magnified image represents the bee-shaped topography on the surfaces. (b) Surface when the hydrophobic surface is coated with SBS or composites prepared by altered salts.

temperatures with $\mathrm{NaCl}$ addition into the composite. Despite minor differences in $G^{*}$ values, more distinct decreases were obtained in $\delta$ values at low temperatures with composite incorporation into the hydrophobic fluid compared to that of unmodified hydrophobic fluid (Figure 5B). Decrease of $\delta$ at low temperatures is an indication of improved elasticity, which 
(a)

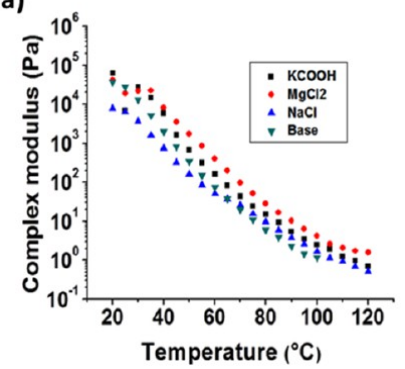

(b)

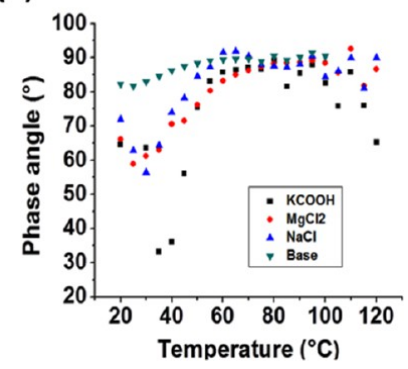

Figure 5. Isochronal plots of (a) complex modulus and (b) phase angle at $0.02 \mathrm{~Hz}$ for the unmodified hydrophobic fluid and ionic salt composite-incorporating hydrophobic fluids.

is a desired property for this particular hydrophobic fluid as elasticity reduces the risk of fracture on surfaces in real-life conditions.

3.5. Anti-icing Property Characterization. In our previous work, we used a temperature- and humiditycontrolled custom-made chamber with $-14{ }^{\circ} \mathrm{C}$ plate, with $5-6{ }^{\circ} \mathrm{C}$ medium temperature and $50 \%$ humidity conditions. ${ }^{26}$ Despite these challenging conditions, we could observe significant delays in freezing on modified hydrophobic surfaces compared to those on unmodified surfaces. In our current study, we conducted freezing experiments in a temperaturecontrolled large chamber that operated similar to realistic ambient conditions during winter. This cold chamber was integrated with a camera where the temperature was maintained at $-2{ }^{\circ} \mathrm{C}$ and uniformly distributed. Freezing delays on surfaces coated with the unmodified hydrophobic fluid and composite-modified hydrophobic fluids were investigated by observing the freezing rate of a water droplet on the surface (Figure 6). Water droplets $(50 \mu \mathrm{L})$ were placed onto each hydrophobic surface, and all samples were kept in the cold chamber. The temperature inside the chamber was also recorded with thermocouples placed in different parts of the chamber. The freezing of droplets was monitored with a camera-attached system and recorded. The freezing time is determined based on the disappearance of transparency of the droplets.

Investigation of the freezing rate of a water droplet on each surface was repeated twice sequentially. Water droplets were left on the surface after each exposure and subsequently evaporated so that ionic salt would not be washed away with water, and new water droplets were then added onto the surface for the second exposure measurements.

Freezing delays on surfaces with unmodified, SBS-coated, composite-incorporated, or composite-coated samples are presented in Figure 7. Coating of hydrophobic surfaces with

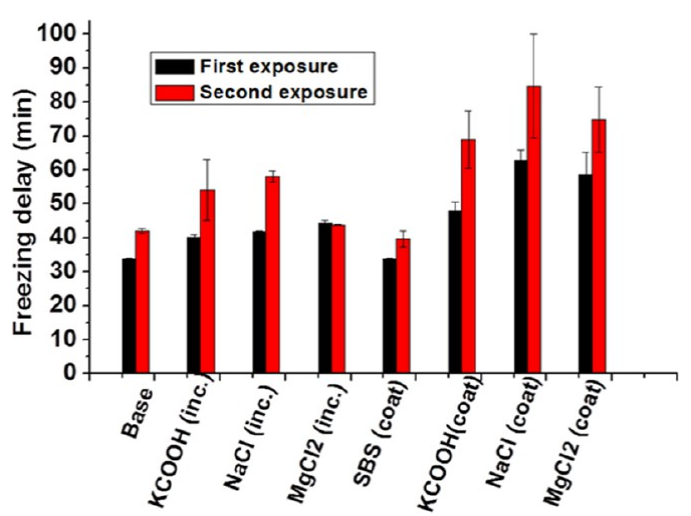

Figure 7. Freezing delays of unmodified, composite-incorporated, and composite-coated hydrophobic surfaces prepared with different salts in the temperature-controlled chamber at $-2{ }^{\circ} \mathrm{C}$.

the SBS polymer did not have a significant influence on the delay of freezing. Higher freezing delays were observed on composite-coated hydrophobic surfaces compared to those on surfaces with composite incorporation. This result was expected, as water droplets were directly in contact with the composite layer, which included encapsulated salt molecules. A higher amount of salt could diffuse out from the composite layer to water droplets on composite-coated samples compared to that on composite-incorporated samples. Thus, longer delays in the freezing were observed on composite-coated hydrophobic surfaces compared to those on surfaces prepared with composite-incorporating hydrophobic surfaces. When we compare the effects of altered ionic salts in composite-coated
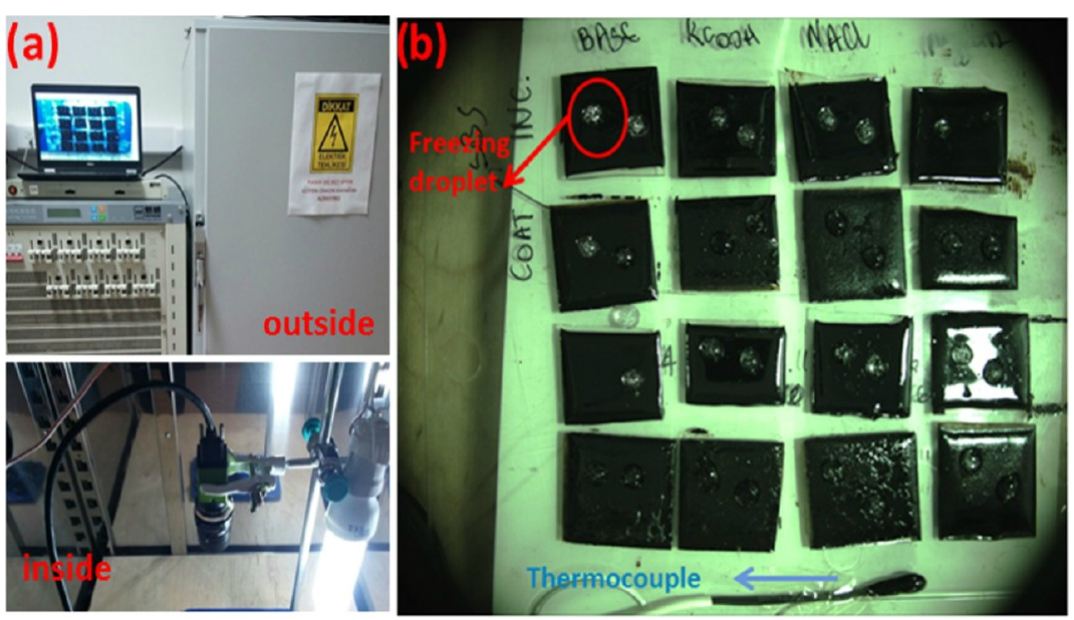

Figure 6. Monitoring of freezing experiments in the cold chamber at $-2{ }^{\circ} \mathrm{C}$. (a) Photographic image of the camera-attached cold chamber where samples inside the chamber were observed on the computer screen. The left bottom image shows inside of the chamber with camera attachment. (b) Freezing of water droplets on hydrophobic surface (unmodified) and surfaces prepared with different salt composite-incorporated hydrophobic surfaces in the cold chamber. 
samples, the highest freezing delay was recorded on the $\mathrm{NaCl}$ containing composite-coated sample. This delay was consistent with the freezing point depression values of the dispersed phase in parenting emulsions, where the same amount of salt was used for each case. The degree in the depression of freezing point of water when an ionic salt is used can be calculated by the following equation

$$
\Delta T_{\mathrm{F}}=K_{\mathrm{F}} \cdot b \cdot i
$$

where $\Delta T_{\mathrm{F}}$ is the amount of decrease in the freezing temperature $\left({ }^{\circ} \mathrm{C}\right) ; K_{\mathrm{F}}$ is the cryoscopic constant, which is an intrinsic property of the solute; $b$ is the molality of the salt solution $(\mathrm{mol} / \mathrm{kg})$; and $i$ is the van't Hoff factor, which is the number of ions per solute molecule. According to this expression, the freezing point depression performance of a particular ionic salt in water is directly proportional to the concentration of ionic salt and ionization degree of the salt.

On the basis of eq 1, the values presented in Table 1 were obtained for the ionic salts used in our study. We expected to

Table 1. Theoretical Amount of Decreases in Freezing Points Based on 1 for the Aqueous Phase in Emulsions Prepared with $0.3 \mathrm{~g} / \mathrm{mL}$ Concentration of Ionic Salt

$\begin{array}{lcccc}\text { ionic salt } & K_{\mathrm{f}}\left({ }^{\circ} \mathrm{C} \mathrm{kg} / \mathrm{mol}\right) & b(\mathrm{~mol} / \mathrm{kg}) & i & \Delta T\left({ }^{\circ} \mathrm{C}\right) \\ \mathrm{KCOOH} & 1.86 & 3.566 & 2 & 13.26 \\ \mathrm{NaCl} & 1.86 & 5.137 & 2 & 19.11 \\ \mathrm{MgCl}_{2} & 1.86 & 3.151 & 3 & 17.58\end{array}$

observe longest delays in the freezing of water on surfaces that included the $\mathrm{NaCl}$ ionic salt in the composite. However, in our experiments, this effect was not observed in compositeincorporated samples, probably due to the differences in the distribution and diffusion properties of ionic salts in the hydrophobic fluid.

Freezing tests on surfaces were performed twice with the same samples consecutively to demonstrate the effects of the repeated freezing process on the icing property of surfaces. After the first freezing test, which was defined as "first exposure", water droplets were kept on the surface and evaporated. Once the surfaces were fully dry after 3 days, new water drops were placed on surfaces for the second exposure. Hence, up to $1 \mathrm{~h}$, freezing delay occurs as a result of ionic salt release for 3 days. Similarly, this salt release will continue even in wetting/drying cycles in following tens of days in realistic conditions, which will provide continued delays in freezing. This continued salt release is also demonstrated in Figure 8 where ionic salt release was measured up to 118 days.

We also tested freezing delays of composite-modified hydrophobic fluid samples with larger sizes prepared in Petri dishes (Figures S2 and S3, Supporting Information) using 6 $\mathrm{mL}$ of water films and a lower freezing test temperature, -8 ${ }^{\circ} \mathrm{C}$, where the larger amount of water takes a longer time to freeze and provides more detailed comparison among altered surfaces. We observed higher freezing delays for surfaces prepared with $\mathrm{KCOOH}$ (Figure S4). This observation is also consistent with our earlier experiments where the amount of $\mathrm{KCOOH}$ release is higher than that of $\mathrm{NaCl}$ or $\mathrm{MgCl}_{2}$ after a week-long incubation of samples in water (Figure 8).

In addition to anti-icing experiments, ice adhesion tests were performed to determine the adhesion strength of ice on modified and unmodified surfaces. Removal of ice from modified surfaces by centrifugal force is a commonly applied

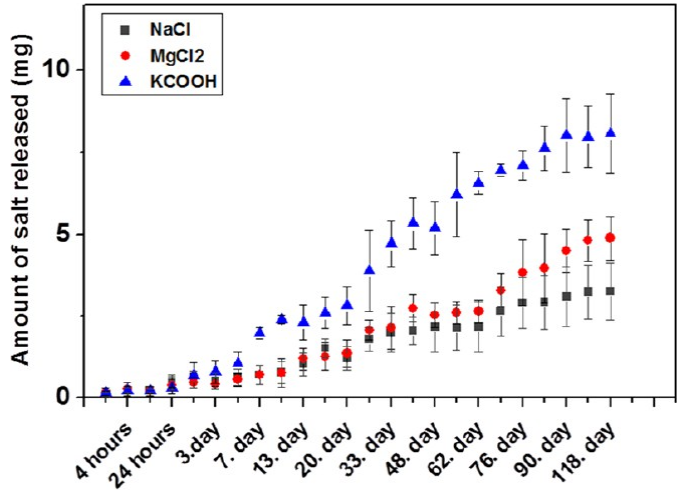

Figure 8. Time-dependent release of $\mathrm{KCOOH}, \mathrm{NaCl}$, and $\mathrm{MgCl}_{2}$ from surfaces prepared with different salt composite-incorporated hydrophobic fluids.

method for the assessment of ice adhesion. ${ }^{34}$ To test the ice adhesion strength, we used centrifugal force at $1000 \mathrm{rpm}$ and recorded the time for the detachment of ice droplets from the surface. Whereas the unmodified surface could keep the ice on its surface for $15.5 \mathrm{~s}$, ice detached earlier from salt compositeincorporated samples (Figure S5). The early detachment of ice from the polymer composite-incorporated samples is the result of a decreased ice adhesion force between the surface and the ice in the presence of an anti-icing component. ${ }^{25}$ On the other hand, for the coated samples, ice detachment was observed later at $22 \mathrm{~s}$ for $\mathrm{KCOOH}$ and $\mathrm{NaCl}$ composite-coated samples. However, for $\mathrm{MgCl}_{2}$ composite-coated samples, ice detachment was not observed. This is attributed to the interaction of the $\mathrm{MgCl}_{2}$ composite with the water layer at the ice-coating interphase. Because of this reason, we did not include $\mathrm{MgCl}_{2}$ coated samples in our ice adhesion figure (Figure S5). When the surface is coated with the polymer composite, water droplets placed on the surface can interact with the hydrophilic domains of the polymer. This results in direct melting of the frozen water droplet on the surface rather than detachment from the surface as a solid entity. Previous research findings show that increased roughness results in increased ice adhesion. $^{35}$ Because the amount of polymer composite is much larger on the surface for the coated samples, surface roughness is also enhanced. Therefore, ice adhesion at the interface of ice and composite is reinforced.

3.6. Characterization of Ionic Salt Release from Functional Surfaces. The release of $\mathrm{KCOOH}, \mathrm{NaCl}$, and $\mathrm{MgCl}_{2}$ from composite-modified hydrophobic surfaces was investigated by placing surfaces in water and measuring the $\mathrm{Cl}^{-}$ and $\mathrm{K}^{+}$ions using ion-selective electrodes. $\mathrm{Cl}^{-}$and $\mathrm{K}^{+}$ion concentrations were measured at specific time points, and the amount of salts released was calculated from the measured values of $\mathrm{Cl}^{-}$and $\mathrm{K}^{+}$concentrations. The amount of ionic salt released at specific time points is shown in Figure 8. We observed that salt release from composite-modified hydrophobic surfaces reached saturation after 118 days. This result shows that freezing delays on composite-incorporated hydrophobic surfaces are expected to increase after 118 days because of the accumulation of salts on the surface. However, significant increase is not expected in the case of compositecoated hydrophobic surfaces because there is no continuous salt diffusion from underlying layers of hydrophobic medium. The highest amount of salt release was observed from the $\mathrm{KCOOH}$-containing surface after 118 days. This can be 
explained by the two possible reasons: (1) $\mathrm{KCOOH}$ was incorporated within the composite in the hydrophobic fluid uniformly and (2) compared to those of other salts, $\mathrm{KCOOH}$ diffusion rates in the hydrophobic fluid were higher. This result shows that the addition of $\mathrm{KCOOH}$ in the compositeincorporated hydrophobic fluid can result in better anti-icing performance compared to that of the other ionic salts for long periods of time.

\section{CONCLUSIONS}

In this study, we investigated anti-icing properties of different ionic salts in composite-modified hydrophobic fluids. We both incorporated composites into hydrophobic fluids and coated hydrophobic surfaces with our composite to investigate and compare the performance of delayed icing properties of alternative approaches. We observed higher delays in the freezing on composite-coated samples compared to those observed on composite-incorporating surfaces. The samples modified with composites that contained $\mathrm{NaCl}$ resulted in higher delays in freezing when concentrations of ionic salts were kept constant. These results suggested that delays in freezing could be tuned with the type and concentration of the anti-icing agent incorporation into the composite.

We also investigated the effect of particular ionic salt on the mechanical and morphological properties of the compositeincorporated or -coated hydrophobic surfaces. We obtained stable emulsions and surfaces with uniform morphologies when composites were prepared with $\mathrm{KCOOH}$ or $\mathrm{MgCl}_{2}$. The complex modulus of hydrophobic fluid was not affected significantly with the type of ionic salt. On the other hand, composite-modified hydrophobic fluids showed improved elastic properties. A comparison of ionic salts to delay ice formation on surfaces demonstrated the possibilities and potential of using alternative salts for inducing anti-icing behavior on surfaces. Comparison of the application methods of the anti-icing agents into the hydrophobic fluids (surface incorporation or surface coating) suggested that desirable delays in freezing could be achieved with the most appropriate approach.

\section{ASSOCIATED CONTENT}

\section{S Supporting Information}

The Supporting Information is available free of charge on the ACS Publications website at DOI: 10.1021/acsomega.8b00816.

SEM image of the unmodified hydrophobic surface; images of freezing of water films on hydrophobic surfaces prepared with different salt composite-incorporated hydrophobic surfaces in the cold chamber; schematics for the preparation of composite-incorporating and composite-coated Petri dish samples and freezing experiments; plots and discussions of freezing delays of Petri dish samples of unmodified, compositeincorporated, and composite-coated hydrophobic surfaces in the temperature-controlled chamber at $-8{ }^{\circ} \mathrm{C}$; plots of ice removal times from unmodified, compositeincorporated, and composite-coated samples (PDF)

\section{AUTHOR INFORMATION}

\section{Corresponding Author}

*E-mail: skizilel@ku.edu.tr. Fax: +90-212-338-1548.

\section{ORCID}

Seda Kizilel: 0000-0001-9092-2698

\section{Notes}

The authors declare no competing financial interest.

\section{ACKNOWLEDGMENTS}

The authors acknowledge the financial support from GENERALI and Turkish Petroleum Refineries Corporation (TÜPRAŞ). AFM and SEM characterization experiments were performed at Koç University Surface Science and Technology Center (KUYTAM). Anti-icing property characterization experiments were conducted at Koç University TÜPRAŞ Energy Center (KUTEM). We would like to thank Vahid Pourreza Ghoushchi for his help in camera setup for freezing experiments.

\section{REFERENCES}

(1) Strong, C. K.; Ye, Z.; Shi, X. Safety effects of winter weather: the state of knowledge and remaining challenges. Transp. Rev. 2010, 30, 677-699.

(2) Liu, X.; Wu, S. Research on the conductive asphalt concrete's piezoresistivity effect and its mechanism. Constr. Build. Mater. 2009, 23, 2752-2756.

(3) Ganjyal, G.; Fang, Q.; Hanna, M. A. Freezing points and smallscale deicing tests for salts of levulinic acid made from grain sorghum. Bioresour. Technol. 2007, 98, 2814-2818.

(4) Zhang, S.; Huang, J.; Cheng, Y.; Yang, H.; Chen, Z.; Lai, Y. Bioinspired Surfaces with Superwettability for Anti-Icing and IcePhobic Application: Concept, Mechanism, and Design. Small 2017, 13, No. 1701867.

(5) Liu, X.; Wu, S. Study on the graphite and carbon fiber modified asphalt concrete. Constr. Build. Mater. 2011, 25, 1807-1811.

(6) Sato, K.; Hori, H. Development of Anti-icing Asphalt Pavements Using Rubber Particles, Paper presented at: New Challenges for Winter Road Service, XIth International Winter Road Congress, 2002.

(7) Lund, J. W. Reconstruction of a pavement geothermal deicing system. Geo-Heat Cent. Q. Bull. 1999, 20, 14-17.

(8) Emelyanenko, A. M.; Boinovich, L. B.; Bezdomnikov, A. A.; Chulkova, E. V.; Emelyanenko, K. A. Reinforced superhydrophobic coating on silicone rubber for longstanding anti-icing performance in severe conditions. ACS Appl. Mater. Interfaces 2017, 9, 24210-24219.

(9) Chernyy, S.; Jarn, M.; Shimizu, K.; et al. Superhydrophilic polyelectrolyte brush layers with imparted anti-icing properties: effect of counter ions. ACS Appl. Mater. Interfaces 2014, 6, 6487-6496.

(10) Zhang, Y.; Anim-Danso, E.; Bekele, S.; Dhinojwala, A. Effect of surface energy on freezing temperature of water. ACS Appl. Mater. Interfaces 2016, 8, 17583-17590.

(11) Lai, Y.; Huang, J.; Cui, Z.; et al. Recent Advances in $\mathrm{TiO}_{2-}$ Based Nanostructured Surfaces with Controllable Wettability and Adhesion. Small 2016, 12, 2203-2224.

(12) Shen, Y.; Wang, G.; Tao, J.; et al. Anti-Icing Performance of Superhydrophobic Texture Surfaces Depending on Reference Environments. Adv. Mater. Interfaces 2017, 4, No. 1700836.

(13) Wang, L.; Gong, Q.; Zhan, S.; Jiang, L.; Zheng, Y. Robust AntiIcing Performance of a Flexible Superhydrophobic Surface. Adv. Mater. 2016, 28, 7729-7735.

(14) Kreder, M. J.; Alvarenga, J.; Kim, P.; Aizenberg, J. Design of anti-icing surfaces: smooth, textured or slippery? Nat. Rev. Mater. 2016, 1, No. 15003.

(15) Wong, T.-S.; Kang, S. H.; Tang, S. K.; et al. Bioinspired selfrepairing slippery surfaces with pressure-stable omniphobicity. Nature 2011, 477, 443

(16) Chen, J.; Dou, R.; Cui, D.; et al. Robust prototypical anti-icing coatings with a self-lubricating liquid water layer between ice and substrate. ACS Appl. Mater. Interfaces 2013, 5, 4026-4030.

(17) Kim, P.; Wong, T.-S.; Alvarenga, J.; Kreder, M. J.; AdornoMartinez, W. E.; Aizenberg, J. Liquid-infused nanostructured surfaces 
with extreme anti-ice and anti-frost performance. ACS Nano 2012, 6, 6569-6577.

(18) Chen, J.; Luo, Z.; Fan, Q.; Lv, J.; Wang, J. Anti-Ice Coating Inspired by Ice Skating. Small 2014, 10, 4693-4699.

(19) Rothstein, J. P. Slip on superhydrophobic surfaces. Annu. Rev. Fluid Mech. 2010, 42, 89-109.

(20) Varanasi, K. K.; Deng, T.; Smith, J. D.; Hsu, M.; Bhate, N. Frost formation and ice adhesion on superhydrophobic surfaces. Appl. Phys. Lett. 2010, 97, No. 234102.

(21) Kulinich, S. A.; Farhadi, S.; Nose, K.; Du, X. W. Superhydrophobic Surfaces: Are They Really Ice-Repellent? Langmuir 2011, 27, 25-29.

(22) Mauro, A.; Grossman, J. C. Street-heat: Controlling road temperature via low enthalpy geothermal energy. Appl. Therm. Eng. 2017, 110, 1653-1658.

(23) Cao, L.; Jones, A. K.; Sikka, V. K.; Wu, J.; Gao, D. Anti-icing superhydrophobic coatings. Langmuir 2009, 25, 12444-12448.

(24) Liu, Z.; Xing, M.; Chen, S.; He, R.; Cong, P. Influence of the chloride-based anti-freeze filler on the properties of asphalt mixtures. Constr. Build. Mater. 2014, 51, 133-140.

(25) Giuliani, F.; Merusi, F.; Polacco, G.; Filippi, S.; Paci, M. Effectiveness of sodium chloride-based anti-icing filler in asphalt mixtures. Constr. Build. Mater. 2012, 30, 174-179.

(26) Aydın, D.; Kizilel, R.; Caniaz, R. O.; Kizilel, S. GelationStabilized Functional Composite-Modified Bitumen for Anti-icing Purposes. Ind. Eng. Chem. Res. 2015, 54, 12587-12596.

(27) Kanyas, S.; Aydin, D.; Kizilel, R.; Demirel, A. L.; Kizilel, S. Nanoparticle and gelation stabilized functional composites of an ionic salt in a hydrophobic polymer matrix. PLoS One 2014, 9, No. e88125.

(28) Adubiaro, H.; Olaofe, O.; Akintayo, E. Effect of Salts on the Emulsifying Properties of Adansonia digitata (Baobab) Seed Flour. Adv. J. Food Sci. Technol. 2012, 4, 126-129.

(29) Binks, B. P.; Murakami, R.; Armes, S. P.; Fujii, S. Effects of pH and salt concentration on oil-in-water emulsions stabilized solely by nanocomposite microgel particles. Langmuir 2006, 22, 2050-2057.

(30) Jo, Y.-J.; Kwon, Y.-J.; Min, S.-G.; Choi, M.-J. Effect of $\mathrm{NaCl}$ Concentration on the Emulsifying Properties of Myofibrilla Protein in the Soybean Oil and Fish Oil Emulsion. Korean J. Food Sci. Anim. Resour. 2015, 35, 315.

(31) Dourado, E. R.; Simao, R. A.; Leite, L. F. M. Mechanical properties of asphalt binders evaluated by atomic force microscopy. $J$. Microsc. 2012, 245, 119-128.

(32) Jäger, A.; Lackner, R.; Eisenmenger-Sittner, C.; Blab, R. Identification of microstructural components of bitumen by means of atomic force microscopy (AFM). PAMM 2004, 4, 400-401.

(33) Airey, G. Rheological properties of styrene butadiene styrene polymer modified road bitumens. Fuel 2003, 82, 1709-1719.

(34) Farhadi, S.; Farzaneh, M.; Kulinich, S. Anti-icing performance of superhydrophobic surfaces. Appl. Surf. Sci. 2011, 257, 6264-6269.

(35) Wang, C.; Zhang, W.; Siva, A.; Tiea, D.; Wynne, K. J. Laboratory test for ice adhesion strength using commercial instrumentation. Langmuir 2014, 30, 540-547. 